\title{
THE FUNCTIONAL EQUATION OF SOME DIRICHLET SERIES. II
}

\author{
BRUCE C. BERNDT
}

Abstract. We derive the functional equation of a class of Dirichlet series. A particular case of our result was first given by Rademacher.

For any positive integer $k$, Rademacher [2] showed that the Dirichlet series

$$
Z(s)=\sum_{l=1}^{k}\left\{\sum_{n>0 ; n \equiv l(k)} n^{-s}+\sum_{n>0 ; n \equiv-l(k)} n^{-s}\right\}^{2} \quad(\sigma=\operatorname{Re} s>1)
$$

has an analytic continuation to the entire complex $s$-plane that is analytic except for a double pole at $s=1$, and satisfies the functional equation

$$
(\pi / k)^{-s} \Gamma^{2}(s / 2) Z(s)=(\pi / k)^{s-1} \Gamma^{2}(\{1-s\} / 2) Z(1-s) .
$$

Rademacher's proof used a familiar representation of the Hurwitz zetafunction. The purpose of this note is to show that a simpler proof of (1) as well as a considerable generalization can be given by employing Epstein zeta-functions rather than the Hurwitz zeta-function.

For $g$ and $h$ real and $\sigma>1$ let

$$
Z(s ; g, h)=\sum_{n}^{\prime} e^{2 \pi i h n}|n+g|^{-s},
$$

where the dash ' indicates that the summation is over all integers $n$ except in the possibility that $n+g=0 . Z(s ; g, h)$ has an analytic continuation to the entire complex plane and is entire if $h$ is not an integer and is analytic everywhere except at $s=1$ where there is a simple pole with residue 2 when $h$ is an integer [1]. Furthermore, [1, p. 207] we have the functional equation

$$
\pi^{-s / 2} \Gamma(s / 2) Z(s ; g, h)=e^{-2 \pi i g h} \pi^{(s-1) / 2} \Gamma(\{1-s\} / 2) Z(1-s ; h,-g) .
$$

Received by the editors November 13, 1970.

AMS 1969 subject classifications. Primary 1041.

Key words and phrases. Dirichlet series, functional equation, Epstein zeta-function, Hurwitz zeta-function. 
Now, for any fixed positive integer $k$ and $h$ real, define for all $s$,

and

$$
Z_{h}(s)=\sum_{l=1}^{k} e^{4 \pi i l h / k} Z^{2}(s ; l / k, h)
$$

$$
Z_{h}^{*}(s)=\sum_{l=1}^{k} Z(s ;(l+h) / k, 0) Z(s ;(k-l+h) / k, 0) .
$$

We shall now prove the

THeOREM. $Z_{h}(s)$ and $Z_{h}^{*}(s)$ satisfy the functional equation

$$
(\pi k)^{-s} \Gamma^{2}(s / 2) Z_{h}(s)=(\pi k)^{s-1} \Gamma^{2}(\{1-s\} / 2) Z_{h}^{*}(1-s) .
$$

For all $h, Z_{h}^{*}(s)$ has a double pole at $s=1$. If $k$ is even and $h \equiv 0\left(\bmod \frac{1}{2} k\right)$, or if $k$ is odd and $h \equiv 0(\bmod k)$, then $Z_{h}(s)$ has a double pole at $s=1$. If $h$ is an integer not satisfying either of the former conditions, $Z_{h}(s)$ is either entire or has a simple pole at $s=1$. If $h$ is not an integer, $Z_{h}(s)$ is entire.

Proof. Consider (2) with $g=l / k, 1 \leqq l \leqq k$, and $\sigma<0$. Put $n=$ $m k+j,-\infty<m<\infty, j=1, \cdots, k$. Then,

$$
\begin{aligned}
\pi^{-s / 2} \Gamma(s / 2) e^{2 \pi i l h / k} Z(s ; l / k, h) \\
\quad=\pi^{(s-1) / 2} \Gamma(\{1-s\} / 2) \sum_{n}^{\prime} e^{-2 \pi i l n / k}|n+h|^{s-1} \\
\quad=\pi^{(s-1) / 2} \Gamma(\{1-s\} / 2) \sum_{j=1}^{k} e^{-2 \pi i l j / k} \sum_{m}^{\prime}|m k+j+h|^{s-1},
\end{aligned}
$$

or

$$
(\pi k)^{-s / 2} \Gamma(s / 2) e^{2 \pi i l h / k} Z(s ; l / k, h)
$$

Define

$$
=(\pi k)^{(s-1) / 2} \Gamma(\{1-s\} / 2) k^{-1 / 2} \sum_{j=1}^{k} e^{-2 \pi i l j / k} Z(1-s ;(j+h) / k, 0) .
$$

$$
\xi(s ; l / k, h)=(\pi k)^{-s / 2} \Gamma(s / 2) e^{2 \pi i l h / k} Z(s ; l / k, h),
$$

the symmetric $k \times k$ matrix $A=\left[a_{l j}\right]=\left[k^{-1 / 2} e^{-2 \pi i l j / k}\right]$, and $v_{r}(s, h)$ to be the column vector whose $l$ th component is $\xi(s ;(l+r) / k, h), 1 \leqq l \leqq k$. Then the $k$ relations given by (4) can be written as

$$
v_{0}(s, h)=A v_{h}(1-s, 0) .
$$

Now, $A^{2}=H=k^{-1}\left[b_{l j}\right]$, where

$$
\begin{aligned}
b_{l j}=\sum_{m=1}^{k} e^{-2 \pi i(l+j) m / k} & =k, & & \text { if } l+j=k \text { or } 2 k, \\
& =0, & & \text { otherwise, }
\end{aligned}
$$


i.e., if $H=\left[h_{l j}\right], h_{l j}=0$ except when $l+j \equiv 0(\bmod k)$ in which case $h_{l j}=1$. If $T$ denotes the transpose, we then have from (5)

$$
\begin{aligned}
& (\pi k)^{-s} \Gamma^{2}(s / 2) Z_{h}(s) \\
& \quad=v_{0}^{T}(s, h) v_{0}(s, h)=\left\{A v_{h}(1-s, 0)\right\}^{T} A v_{h}(1-s, 0) \\
& \quad=v_{h}(1-s, 0)^{T} H v_{h}(1-s, 0)=(\pi k)^{s-1} \Gamma^{2}(\{1-s\} / 2) Z_{h}^{*}(1-s),
\end{aligned}
$$

by a direct calculation and the fact that $Z(s ; h / k, 0)=Z(s ;(h+k) / k, 0)$. This then proves (3).

It is clear from our remarks on $Z(s ; g, h)$ that $Z_{h}^{*}(s)$ has a double pole at $s=1$. Also, if $h \equiv 0\left(\bmod \frac{1}{2} k\right)$ when $k$ is even, or if $h \equiv 0(\bmod k)$ when $k$ is odd, the coefficient of $(s-1)^{-2}$ in the Laurent expansion of $Z_{h}(s)$ about $s=1$ is easily seen to be $4 k$. However, for other integral values of $h$, the coefficient of $(s-1)^{-2}$ is

$$
\sum_{l=1}^{k} 4 e^{2 \pi i l(2 h) / k}=0 .
$$

In general, the constant term in the Laurent expansion of $Z(s ; g, h)$ about $s=1$ is a function of $g$. Thus, $Z_{h}(s)$ may have a simple pole at $s=1$ or might be analytic at $s=1$. Since $Z(s ; g, h)$ is entire if $h$ is not an integer, then clearly $Z_{h}(s)$ is entire as well, and this completes the proof.

We now show that Rademacher's result (1) is a special case of (3). Put $h=0$ in (3). It is readily seen that $Z(s ; l / k, 0)=Z(s ;(k-l) / k, 0)$. Hence $Z_{0}(s)=Z_{0}^{*}(s)$. Now for $\sigma>1$,

$$
\begin{aligned}
Z_{0}(s) & =\sum_{l=1}^{k} Z^{2}(s ; l / k, 0)=k^{2 s} \sum_{l=1}^{k}\left\{\sum_{m}^{\prime}|m k+l|^{-s}\right\}^{2}=k^{2 s} \sum_{l=1}^{k}\left\{\sum_{n \equiv l(k)}^{\prime}|n|^{-s}\right\}^{2} \\
& =k^{2 s} \sum_{l=1}^{k}\left\{\sum_{n>0 ; n=l(k)} n^{-s}+\sum_{n>0 ; n=-l(k)} n^{-s}\right\}^{2}=k^{2 s} Z(s),
\end{aligned}
$$

and hence (3) reduces to (1).

\section{REFERENCES}

1. Paul Epstein, Zur Theorie allgemeiner Zetafunktionen. II, Math. Ann. 63 (1907), 205-216.

2. Hans Rademacher, On the Hurwitz zetafunction, Report of the Institute in the Theory of Numbers, University of Colorado, Boulder, Col., 1959, pp. 73-77.

Department of Mathematics, University of Illinois, Urbana, Illinois 61801 